ARTICLE

Translational Therapeutics

\title{
Targeting glutaminolysis in chondrosarcoma in context of the IDH1/2 mutation
}

\author{
Elisabeth F. P. Peterse ${ }^{1}$, Bertine Niessen ${ }^{1}$, Ruben D. Addie ${ }^{1}$, Yvonne de Jong ${ }^{1}$, Arjen H. G. Cleven ${ }^{1}$, Alwine B. Kruisselbrink ${ }^{1}$,
} Brendy E. W. M. van den Akker ${ }^{1}$, Remco J. Molenaar ${ }^{2}$, Anne-Marie Cleton-Jansen ${ }^{1}$ and Judith V. M. G. Bovée ${ }^{1}$

INTRODUCTION: Chondrosarcoma is a malignant cartilage-forming bone tumour in which mutations in IDH1 and IDH2 frequently occur. Previous studies suggest an increased dependency on glutaminolysis in IDH1/2 mutant cells, which resulted in clinical trials with the drugs CB-839, metformin and chloroquine. In this study, the preclinical rationale for using these drugs as a treatment for chondrosarcoma was evaluated.

METHODS: Expression of glutaminase was determined in 120 cartilage tumours by immunohistochemistry. Ten chondrosarcoma cell lines were treated with the metabolic compounds CB-849, metformin, phenformin (lipophilic analogue of metformin) and chloroquine.

RESULTS: A difference in glutaminase expression levels between the different tumour grades $(p=0.001$, one-way ANOVA) was identified, with the highest expression observed in high-grade chondrosarcomas. Treatment with CB-839, metformin, phenformin or chloroquine revealed that chondrosarcoma cell lines are sensitive to glutaminolysis inhibition. Metformin and phenformin decreased mTOR activity in chondrosarcoma cells, and metformin decreased LC3B-II levels, which is counteracted by chloroquine. CONCLUSION: Targeting glutaminolysis with CB-839, metformin, phenformin or chloroquine is a potential therapeutic strategy for a subset of high-grade chondrosarcomas, irrespective of the presence or absence of an IDH1/2 mutation.

British Journal of Cancer https://doi.org/10.1038/s41416-018-0050-9

\section{INTRODUCTION}

Chondrosarcoma is the second most common primary bone malignancy in humans. It represents a heterogeneous collection of cartilage-forming tumours, which can be divided in several subtypes and histological grades. ${ }^{1}$ The most common subtype is conventional chondrosarcoma (85\%), which arises centrally in the medulla of the bone. Conventional chondrosarcoma is histologically graded to determine treatment strategy and the patient's prognosis. The atypical cartilaginous tumour (ACT, previously known as chondrosarcoma grade 1), accounts for $61 \%$ of cases. First-line treatment consists of curettage with local adjuvant treatment, resulting in a 5 -year survival rate of $95 \%$. Grade II (36\%) and grade III (3\%) chondrosarcomas have a worse 5-year survival of $86 \%$ and $58 \%$, respectively, due to the occurrence of metastases. ${ }^{1-3}$ These tumours are treated with en bloc resection. Dedifferentiated chondrosarcoma is a highly malignant subtype with an overall survival rate of $7-24 \% .{ }^{4}$ Mesenchymal chondrosarcoma has a 10 -year survival rate between 44 and $54 \% .^{5,6}$ It is a rare aggressive subtype in which distant metastasis can be identified even after 20 years. $^{5-7}$ Chondrosarcoma patients with inoperable disease, due to tumour location, tumour size or extensive metastatic disease benefit from a doxorubicin-based chemotherapeutic regimen, which increases the 3-year survival from 8 to $26 \% .^{8}$ As the overall efficacy of chemotherapy is limited, new treatment options are needed, which can be identified by further unravelling the essential driver genes and pathways of these tumours.

Potential driver mutations of central conventional and dedifferentiated chondrosarcoma are gain of function mutations in isocitrate dehydrogenase 1 and 2 (IDH1 and IDH2), which have been identified in $38-70 \%$ of the cases. ${ }^{9,10}$ Its occurrence in the benign precursors lesions (enchondromas), of which $52-87 \%$ harbour an IDH1/2 mutation, ${ }^{11,12}$ further demonstrates that IDH1/2 mutations are an early event in chondrosarcoma genesis. IDH1 and IDH2 are essential enzymes in cell metabolism, as they convert isocitrate to a-ketoglutarate $(a-K G)$ in respectively the cytoplasm and the mitochondria. The mutant enzyme acquires the activity to convert $\mathrm{a}-\mathrm{KG}$ to $D$-2-hydroxyglutarate $(D-2-\mathrm{HG})$, an oncometabolite that competitively inhibits the $\mathrm{a}-\mathrm{KG}$ dependent enzymes by the high structural similarities. ${ }^{13}$ Processes involved in chondrosarcoma progression make these cells independent of the mutant IDH enzymes, as treatment with AGI-5198, a specific IDH1 mutant inhibitor, did not influence the tumourigenic properties of these cells. ${ }^{14}$ Therefore, we propose to exploit the metabolic vulnerability caused by the $I D H 1 / 2$ mutations as therapeutic strategy for chondrosarcoma.

IDH1/2 mutant cells need a-KG for the production of $D-2-H G$, which can be generated via glycolysis and glutaminolysis. It has been suggested that IDH1/2 mutated tumours depend on glutaminolysis for their a-KG supply, ${ }^{15-17}$ which led to two clinical

\footnotetext{
${ }^{1}$ Department of Pathology, Leiden University Medical Center, Leiden, The Netherlands and ${ }^{2}$ Department of Medical Biology, Academic Medical Center, University of Amsterdam, Amsterdam, The Netherlands

Correspondence: Judith V. M. G. Bovée (J.V.M.G.bovee@lumc.nl)

These authors contributed equally: Elisabeth F.P. Peterse, Bertine Niessen.
}

Received: 10 November 2017 Revised: 3 February 2018 Accepted: 9 February 2018

Published online: 26 March 2018 


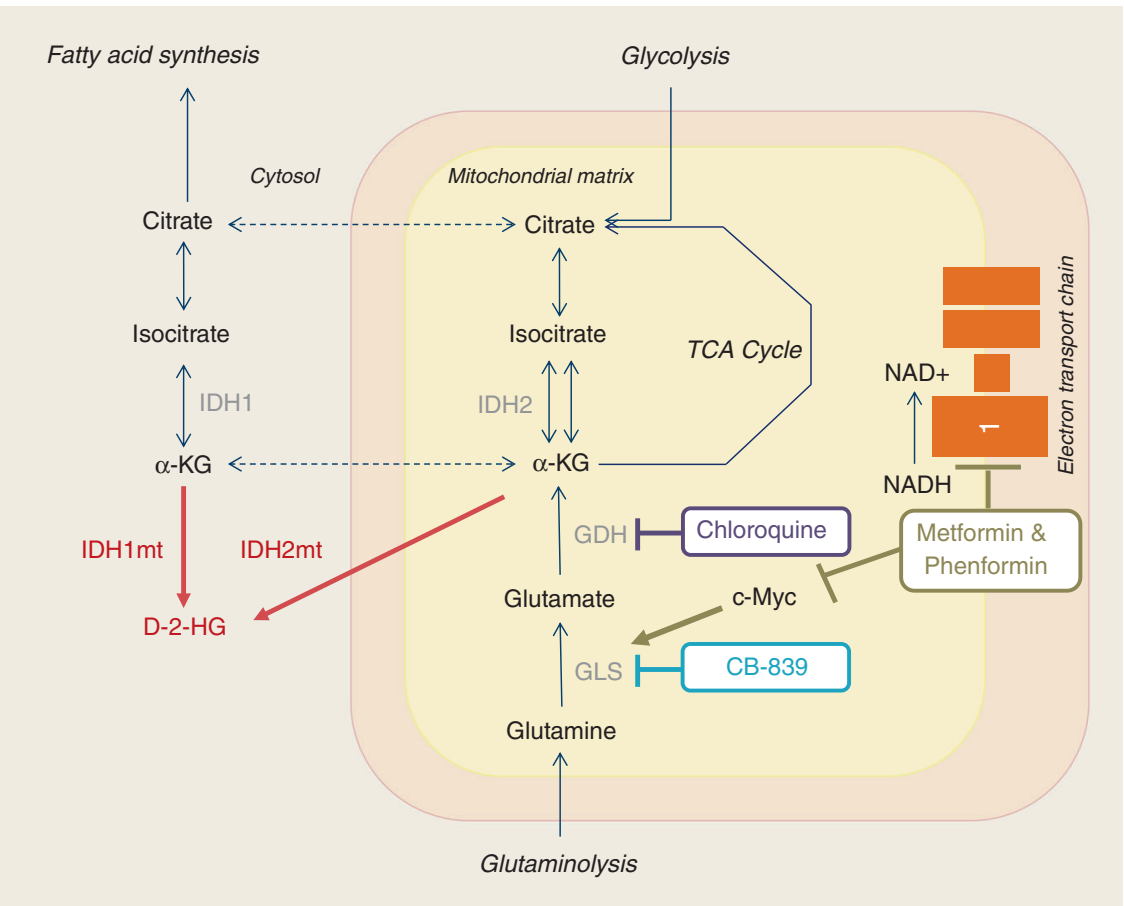

Fig. 1 Schematic representation of glutamine metabolism and the compounds used in this study. IDH isocitrate dehydrogenase, IDHmt mutated IDH, D-2-HG D-2-hydroxyglutarate, $\alpha$-KG $\alpha$-ketoglutarate, GLS glutaminase, GDH glutamate dehydrogenase, NAD nicotinamide adenine dinucleotide, TCA tricarboxylic acid

trials that were recently started in $I D H 1 / 2$ mutated solid tumours, including chondrosarcomas. The first one is a phase I trial with the drug CB-839 (NCT02071862 clinicaltrials.gov), an inhibitor of glutaminase (Fig. 1). The second one is a phase IB/II trial with the drugs metformin and chloroquine (NCT02496741 clinicaltrails.gov), after which the feasibility of phenformin may be explored as an alternative to metformin in case of lack of effect of metformin. ${ }^{18}$ Metformin is a first-in-line drug used for the treatment of type II diabetes mellitus that inhibits gluconeogenesis in the liver. It has several effects on cellular proteins, among which it (1) activates adenosine monophosphate activated protein kinase (AMPK), thereby inhibiting the mammalian target of rapamycin (mTOR); ${ }^{19}$ (2) inhibits complex 1 of the electron transport chain; ${ }^{20}$ and (3) indirectly inhibits glutaminase, the enzyme that converts glutamine to glutamate, via c-Myc; (Fig. 1). ${ }^{21,22}$ Phenformin is a lipophilic analogue of metformin with similar working mechanisms, but in contrast to metformin it does not depend on solute carrier (SLC) 22A1-3 transport to get into cells; ${ }^{20,23}$ The anti-malaria drug chloroquine, in addition to its well-known anti-autophagy potency, is able to inhibit glutamate dehydrogenase, an enzyme converting glutamate to a-KG (Fig. 1). ${ }^{24,25}$

In this study, we evaluate whether there is preclinical rationale to target glutaminolysis as a treatment for chondrosarcoma by determining the expression levels of glutaminase in chondrosarcoma primary tumours and by evaluating the effect of metformin, phenformin, chloroquine and CB-839 on chondrosarcoma cells.

\section{MATERIALS AND METHODS}

Immunohistochemistry on tissue microarrays

Glutaminase monoclonal antibody (AB156776, Abcam) (1:400) was used for immunohistochemical stainings on previously generated and published formalin-fixed, paraffin-embedded tissue microarrays $^{26}$ as described. ${ }^{27}$ Hundred and twenty cartilage tumours could be scored, consisting of 12 benign (enchondromas or osteochondromas), 56 ACTs, 36 grade II and 16 grade III tumours.
Two independently operating observers used the following scoring procedure: intensity score $(0$ negative, 1 weak, 2 moderate, 3 strong $)+$ percentage score $(0=0 \%, 1=1-24 \%, 2$ $=25-49 \%, 3=50-74 \%$ and $4=75-100 \%)$. Discrepancies were discussed to reach consensus. Of the central cartilage lesions that could be scored, the IDH mutation status was known of 54 tumours, of which 33 harboured an IDH1 or IDH2 mutation and 21 were wildtype.

\section{Statistical analysis}

Statistical analysis on immunohistochemistry data was performed using Statistical Package for the Social Sciences 23 (SPSS Statistics, IBM). One-way ANOVA with the Fisher's least significant difference (LSD) post-hoc analysis was used to compare glutaminase expression levels between different tumour grades. The difference in glutaminase protein expression between high-grade cartilage tumours (grade II and grade III cartilage tumours) and low-grade cartilage tumours (enchondromas, osteochondromas and ACT) was determined using independent-samples $T$ test. Results were considered significant at the $a=0.05$ level.

\section{Cell culture}

Five IDH1 or IDH2 mutated (JJ012, ${ }^{28}$ SW1353 (ATCC \#HTB-94),

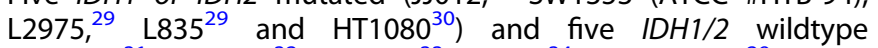

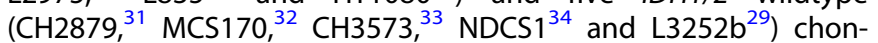
drosarcoma cell lines were analysed. Five of these originate from conventional chondrosarcoma (JJ012, SW1353, L835, CH2879 and CH3573), three from dedifferentiated chondrosarcoma (L2975, NDCS1 and L3252b) and one from mesenchymal chondrosarcoma (MCS170). HT1080 was originally reported as a fibrosarcoma of bone. As this is a diagnosis of exclusion and this cell line is now known to harbour an IDH1 mutation, this tumour most probably reflects a dedifferentiated chondrosarcoma. ${ }^{14}$ Cells were cultured at $5 \% \mathrm{CO}_{2}$ and $37^{\circ} \mathrm{C}$ in a humidified incubator using RPMI 1640 (Gibco) with 10\% (JJ012, SW1353, L2975, HT1080, CH2879 and NDCS1) or $20 \%$ (L835, CH3573 and L3252b) heat inactivated foetal 
A

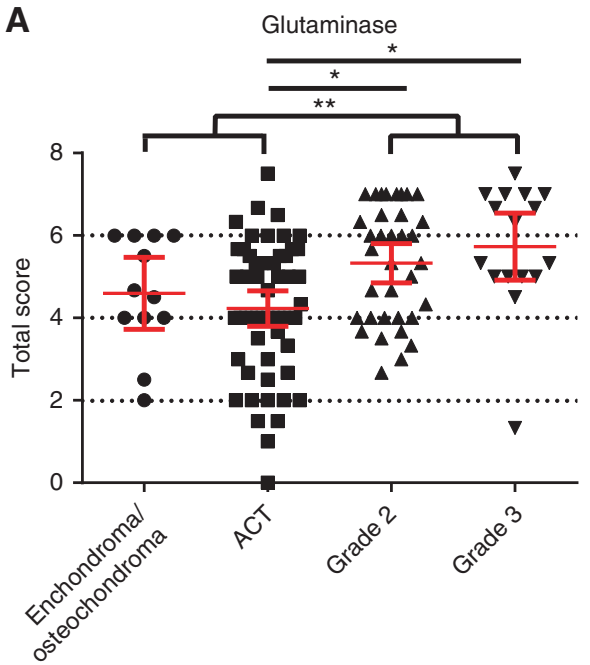

B

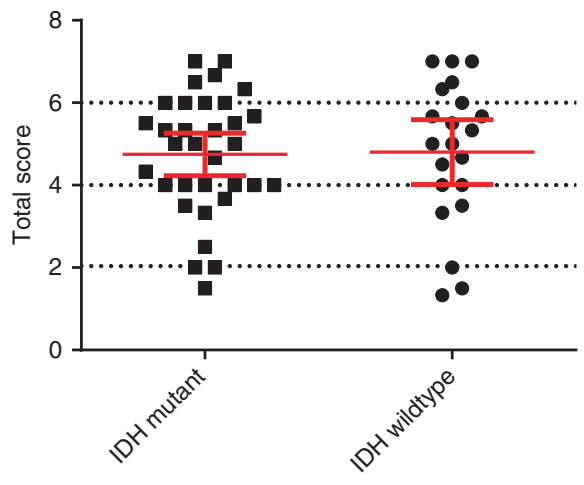

Glutaminase
C

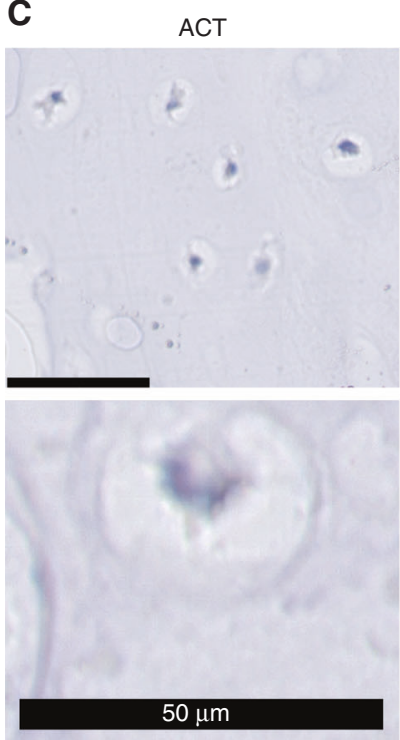

D

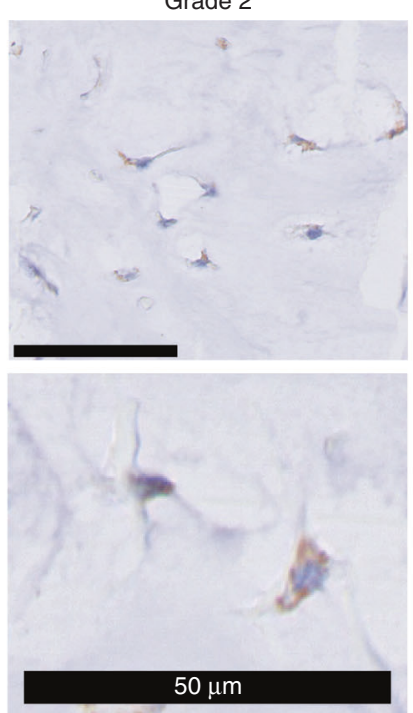


A

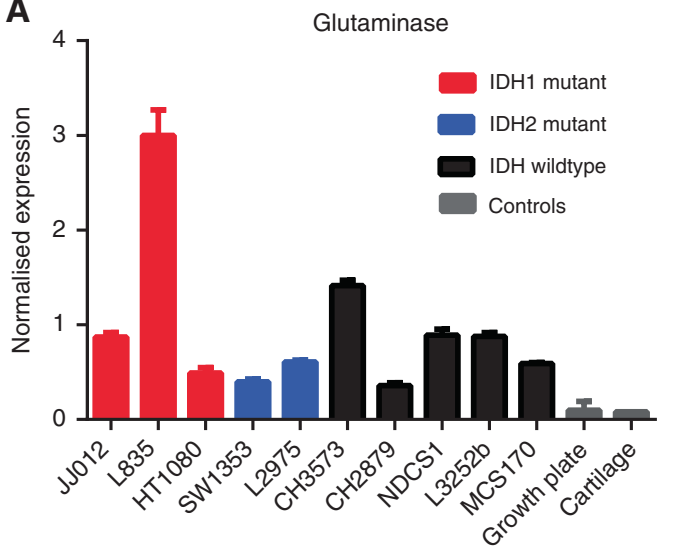

B

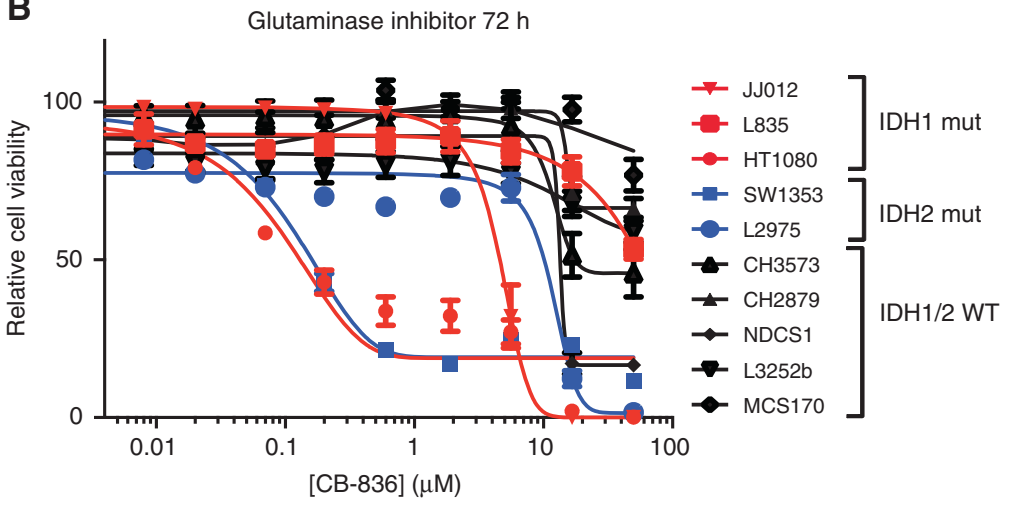

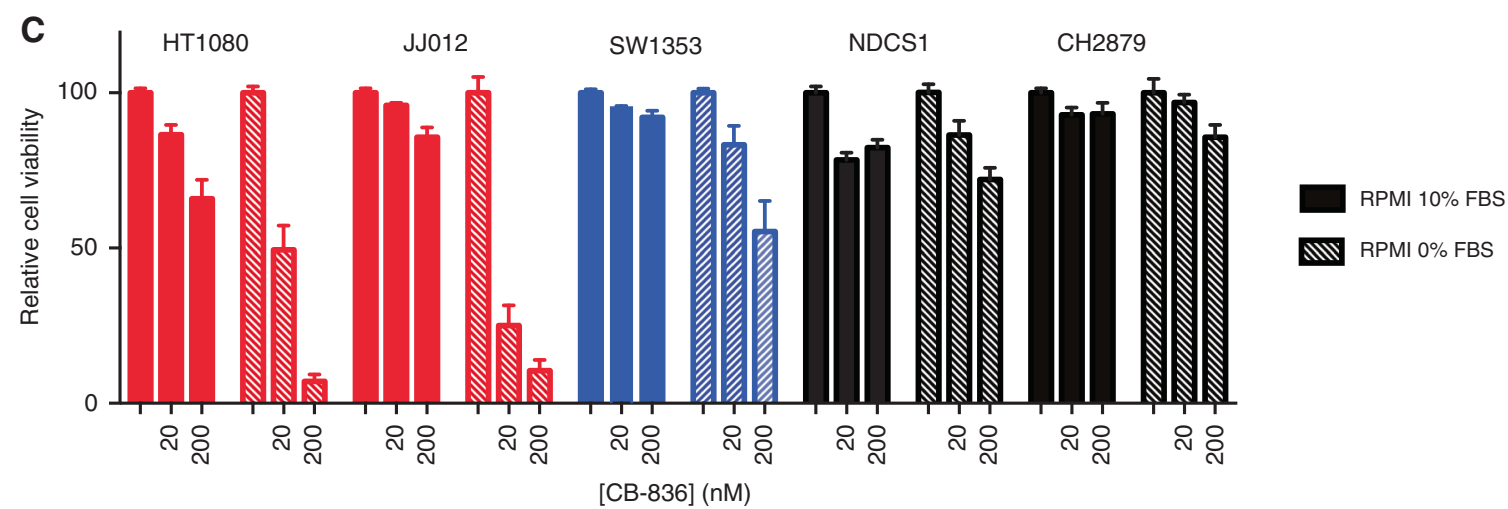

Fig. 3 The glutaminase inhibitor CB-839 inhibits chondrosarcoma cell viability. a Expression levels of glutaminase in ten chondrosarcoma cell lines and two controls. Glutaminase is higher expressed in chondrosarcoma cell lines compared to growth plate and articular cartilage. $\mathbf{b}$ Ten chondrosarcoma cell lines were treated for $72 \mathrm{~h}$ with CB-839, a glutaminase inhibitor. Sensitivity differed between the different cell lines. c Indicated cell lines were treated with 0,20 or $200 \mathrm{nM} \mathrm{CB-839}$ in the presence or absence of FBS. In the absence of FBS, all cell lines are more sensitive for inhibition with CB-839, especially the IDH1/2 mutant cell lines

manufacturer's instructions. Colourimetric values in the plates were subsequently measured using a Wallac 1420 VICTOR2 (Perkin Elmer). Data were analysed in Graphpad Prism 5.0 (www. graphpad.com). For the combination of metformin, phenformin and chloroquine with AGI-5198 (14624, Life technologies), cells were pretreated for $72 \mathrm{~h}$ with AGI-5198 (1 and $10 \mu \mathrm{M})$ or DMSO. For the analyses in which the effect of FBS on CB-839 sensitivity was evaluated, the medium with or without FBS and the corresponding concentrations of the metabolic compounds were added at the same time, so after the cells were allowed to adhere overnight.

\section{Cell count assay}

As the PrestoBlue assay measures mitochondrial activity, we confirmed that the effects of metformin, phenformin and chloroquine on cell viability were caused by an absolute decrease in cell number by fixing the cells in 4\% paraformaldehyde for 15 min, followed by nuclear staining using Hoechst 33342 (Fischer Scientific). The plates were imaged using a BD Pathway 855 imager (Becton Dickinson), after which the images were processed using an Image-Pro Analyser 7.0 algorithm. Hoechst area was used as a read out to quantify the amount of cells in each well.

\section{Analysis of apoptosis}

For analysis of apoptosis, the caspase-glo 3/7 assay (Promega) was used according to manufacturer's instructions. Cells were seeded in white walled 96-wells plates (Corning BV Life Sciences) in densities which resulted in $70 \%$ confluence after $24 \mathrm{~h}$ as described previously. ${ }^{39}$ HT1080, JJ012, SW1353, NDCS1 and CH2879 cells were treated with their $\mathrm{IC}_{75}$ of metformin, phenformin, chloroquine and CB-839 (based on $72 \mathrm{~h}$ dose response curves). The concentration of compounds used was $10 \mathrm{mM}$ metformin, $100 \mu \mathrm{M}$ phenformin, $50 \mu \mathrm{M}$ chloroquine and $6 \mu \mathrm{M}$ CB-839 if $\mathrm{IC}_{75}$, were above these concentrations. $\mathrm{CH} 2879$ cells treated with $1 \mu \mathrm{M}$ doxorubicin (obtained from the in-house hospital pharmacy) and $50 \mu \mathrm{M}$ ABT-737 (Catalog No. S1002, Selleckchem) were used as positive control. For the negative control doxorubicin and ABT-737 were combined with Z-vad-FMK (550377, BD Biosciences). After $24 \mathrm{~h}$ the caspase-glo substrate was added 1:1 followed by incubation of $60 \mathrm{~min}$ at room temperature. Wells were analysed using Wallac 1420 VICTOR2. The experiment was performed two times in duplicate. Data was corrected for plane RPMI control and normalised to untreated control for each cell line. Viability was measured on a simultaneously treated plate after $24 \mathrm{~h}$.

\section{Western blot analysis}

HT1080, JJ012, SW1353, NDCS1 and CH2879 cells are treated with their half maximal inhibitory concentration $\left(\mathrm{IC}_{50}\right)$ values (based on dose response curves of $72 \mathrm{~h}$ ) of metformin, phenformin, chloroquine or CB-839 and lysed after $72 \mathrm{~h}$. A maximum concentration of compounds of $10 \mathrm{mM}$ metformin, $100 \mu \mathrm{M}$ phenformin and $6 \mu \mathrm{M} C \mathrm{CB}-839$ was used if the $\mathrm{IC}_{50}$ was above these concentrations. Western blotting was performed for LC3B (1:1000, clone D11, \#3868, Cell Signaling Technology) and phospho-S6 (1:1000, 2F9, \#4856, Cell Signaling Technology). As a loading control, a-tubulin (1:10,000, clone DM1A, Sigma-Aldrich Chemie) expression was used. Cells were lysed using hot-SDS buffer (1\% SDS, $10 \mathrm{mM}$ Tris/EDTA with complete inhibitor and phosSTOP). For each sample, $10 \mu \mathrm{g}$ protein was loaded on TGX 
Table 1. Absolute $\mathrm{IC}_{50}$ values of $\mathrm{CB}-839$, metformin, phenformin and chloroquine upon $72 \mathrm{~h}$ of treatment, as determined by PrestoBlue Cell Viability

\begin{tabular}{|c|c|c|c|c|c|c|}
\hline Compound/cell line & CB-839 $(\mu \mathrm{M})$ & Metformin (mM) & Phenformin $(\mu \mathrm{M})$ & Chloroquine $(\mu \mathrm{M})$ & IDH1/2 status & Reference \\
\hline נر012 & 4.6 & 19.0 & 504.1 & 27.1 & IDH1 p.Arg132Gly & Scully et al. ${ }^{28}$ \\
\hline SW1353 & 0.2 & 8.26 & 74.5 & 24.6 & IDH2 p.Arg172Ser & ATCC \\
\hline L2975 & 10.2 & 10.6 & 116.6 & 30.5 & IDH2 p.Arg172Trp & Van Oosterwijk et al. ${ }^{29}$ \\
\hline $\mathrm{CH} 2879$ & $>50$ & 10.9 & 193.7 & 57.9 & WT & Gil Benso et al. ${ }^{31}$ \\
\hline $\mathrm{CH} 3573$ & 17.5 & $>20$ & 343.4 & 34.0 & WT & Calabuig-Farinas et al. ${ }^{33}$ \\
\hline L3252b & $>50$ & 16.7 & $>1000$ & 39.4 & WT & Van Oosterwijk et al. ${ }^{29}$ \\
\hline MCS170 & $>50$ & 10.5 & 294.7 & 14.4 & WT & De Jong et al. ${ }^{32}$ \\
\hline
\end{tabular}

A
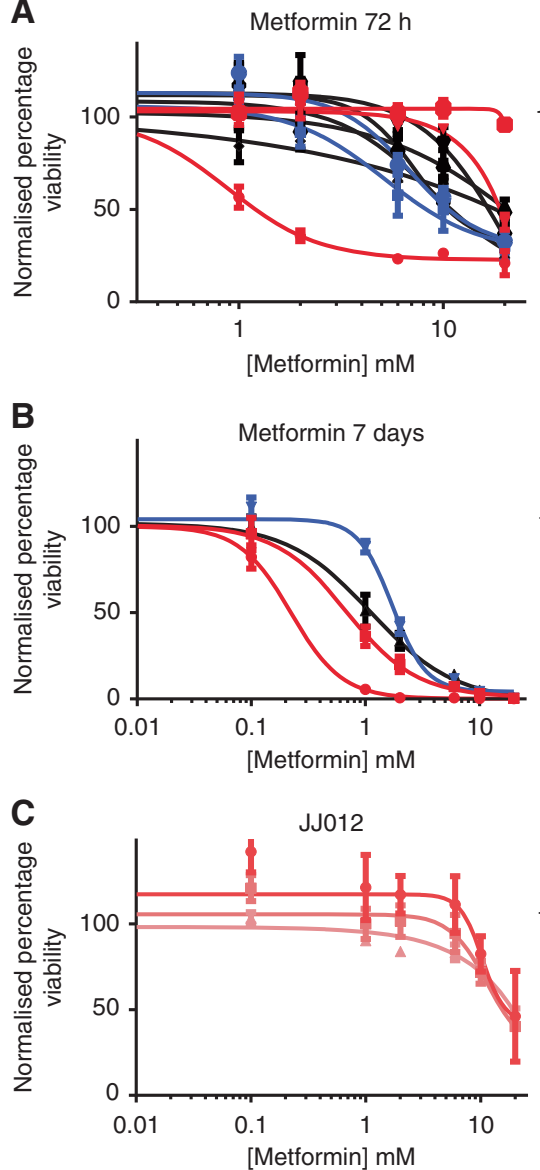

Phenformin $72 \mathrm{~h}$

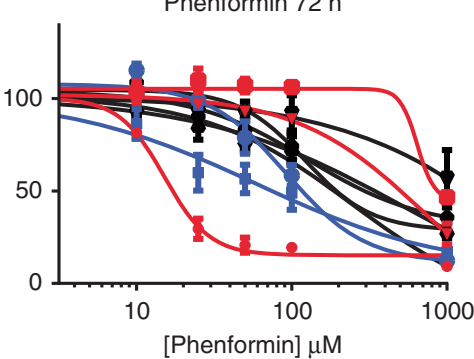

Phenformin 7 days

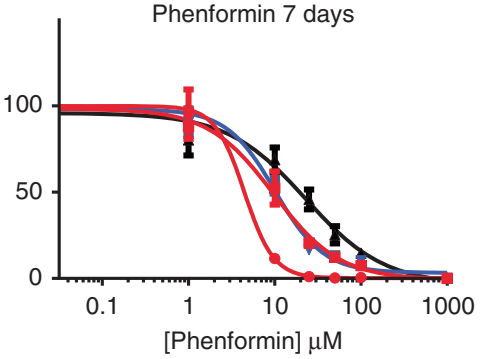

JJ012

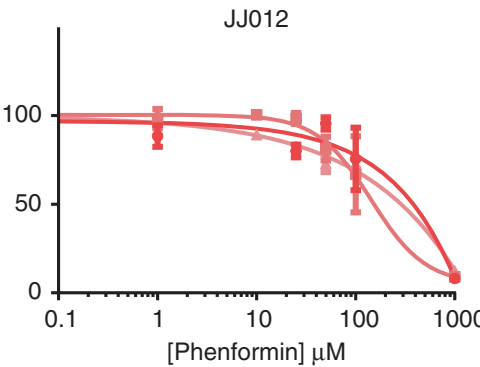

Chloroquine $72 \mathrm{~h}$

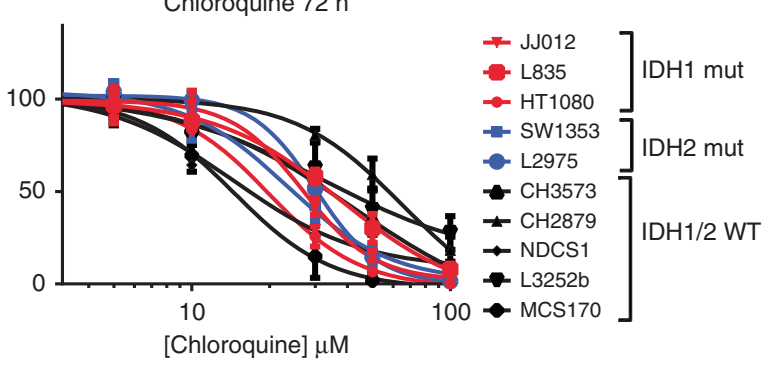

Chloroquine 7 days

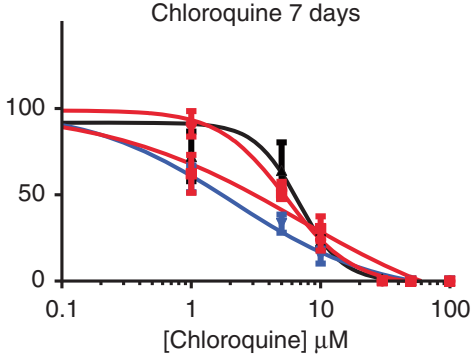

- JJ012

$\rightarrow \mathrm{HT} 1080$

- SW1353

× NDCS1

Fig. 4 Chondrosarcoma cell lines are sensitive for metformin, phenformin and chloroquine, irrespective of the IDH1/2 mutation. a Chondrosarcoma cell lines were treated for $72 \mathrm{~h}$ with corresponding compounds. Cell viability was measured using the PrestoBlue assay. $\mathbf{b}$ Four chondrosarcoma cell lines were treated for 7 days with the corresponding inhibitors. c IDH1 mutant JJ012 cells were pretreated for $72 \mathrm{~h}$ with $10 \mu \mathrm{M} \mathrm{AGI}-5198,1 \mu \mathrm{M}$ AGI-5198 or DMSO, after which they were treated with a combination of AGI-5198 and the corresponding compounds for $72 \mathrm{~h}$. No effect of AGl-5198 was observed

Stain-Free ${ }^{\mathrm{TM}}$ FastCast $^{\mathrm{TM}}$ 12\% Acrylamide Gels (Bio-Rad). Proteins were transferred to a Polyvinylidene difluoride (PVDF) membrane using Trans-Blot ${ }^{\circledR}$ Turbo $^{\mathrm{TM}}$ Transfer System (Bio-Rad) and TransBlot $^{\circledR}$ Turbo $^{\text {TM }}$ RTA Transfer kit PVDF (Bio-Rad) and detected using enhanced chemo-luminescence (Pierce ECL Western Blotting Substrate Fisher Scientific), followed by exposure of $30 \mathrm{~s}$ to 5 min and development of the film (ECL hyperfilm, Amersham, GE Healthcare).
Cell line metabolic profiling

A Seahorse XFe 96 analyser (Seahorse Bioscience, Agilent) was used to measure the oxygen consumption rate (OCR) and the extracellular acidification rate (ECAR) in chondrosarcoma cell lines JJ012, SW1353 and CH2879 after metformin treatment. Thirty hours prior to the assay, cells were plated in optimised densities being 15,000, 13,000 and 30,000 for JJ012, SW1353 and CH2879, respectively. After $6 \mathrm{~h}$ cells were treated with $5 \mathrm{mM}$ metformin for 
$24 \mathrm{~h}$. Before the measurement, cells were incubated for $1 \mathrm{~h}$ in glucose-free RPMI-1640 supplemented with 5\% FBS. During the assay, sequential injections of $10 \mathrm{mM}$ glucose (Sigma-Aldrich), 2.0 $\mu \mathrm{M}$ oligomycin $\mathrm{A}, 2 \mu \mathrm{M}$ carbonyl cyanide-4-(trifluoromethoxy) phenylhydrazone (FCCP) and $0.5 \mu \mathrm{M}$ 1:1 rotenone: antimycin $A$ (Cayman Chemicals) established the metabolic profile of all cell lines. Data was normalised to cell numbers measured in each individual well, determined using a Cellomics HCS fluorescent microscope (Thermoe Fisher) after fixation and Hoechst staining. Data represented as the average \pm SD of triplicate measurements for metformin treated cells and 5-7 replicates for controls.

\section{RESULTS}

Glutaminase is a potential therapeutic target in a subset of chondrosarcomas

By immunohistochemistry, a difference in glutaminase expression levels between the different tumour grades was identified (ANOVA, $p=0.001$ ), and the post-hoc analysis revealed that specifically the grade II tumours and the grade III tumours had higher expression levels compared to the ACTs (both $p=0.001$ ) (Fig. 2a-e). As no difference between central and peripheral cartilage tumours was observed, these were combined in the analyses. Grouping the high-grade cartilage tumours (grade II and

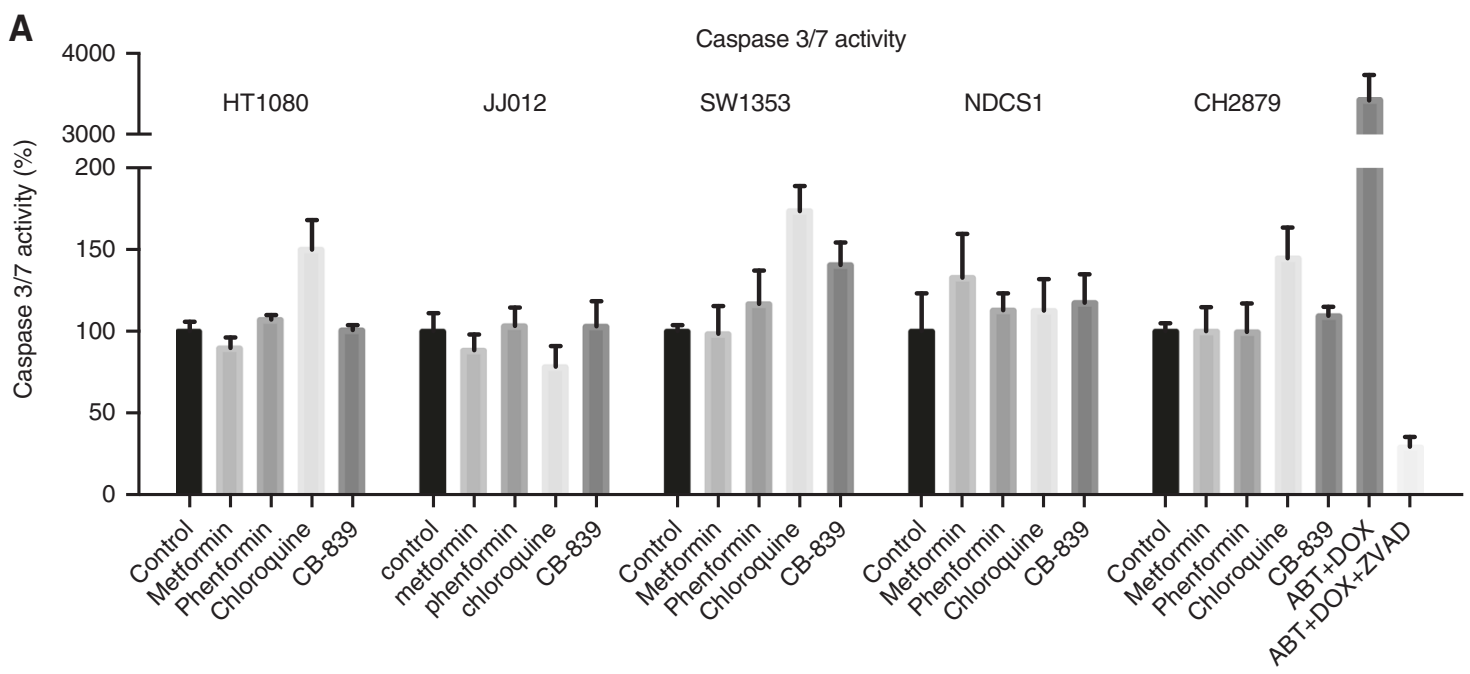

B

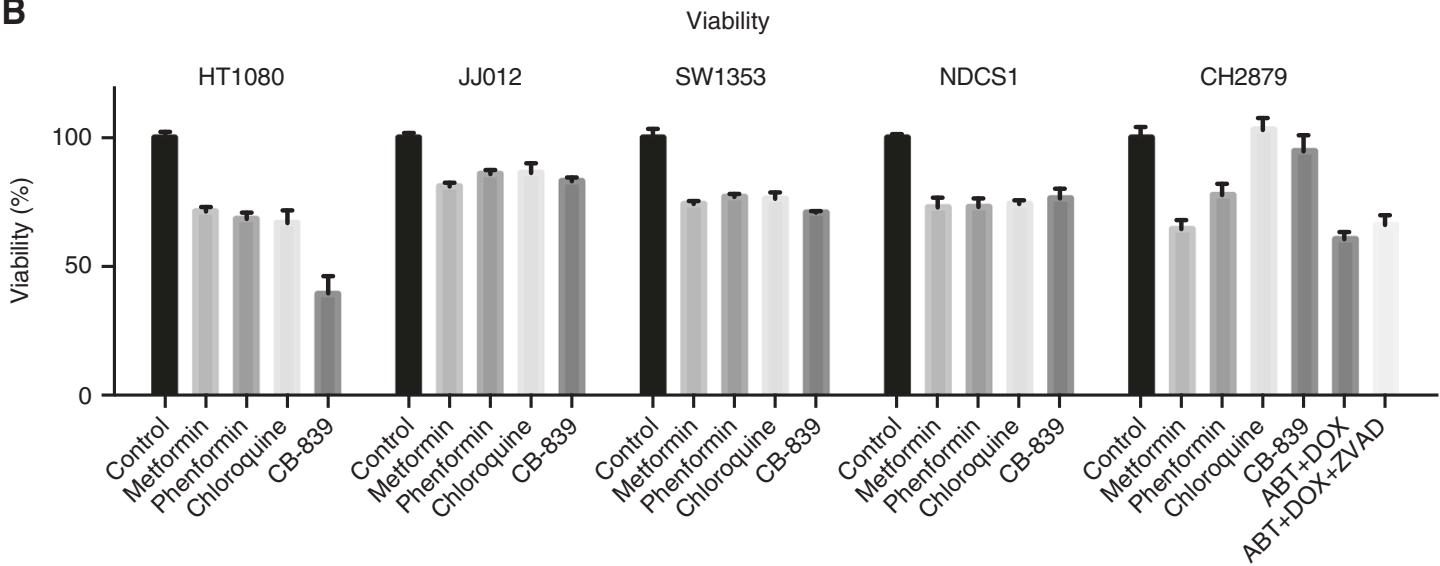

C

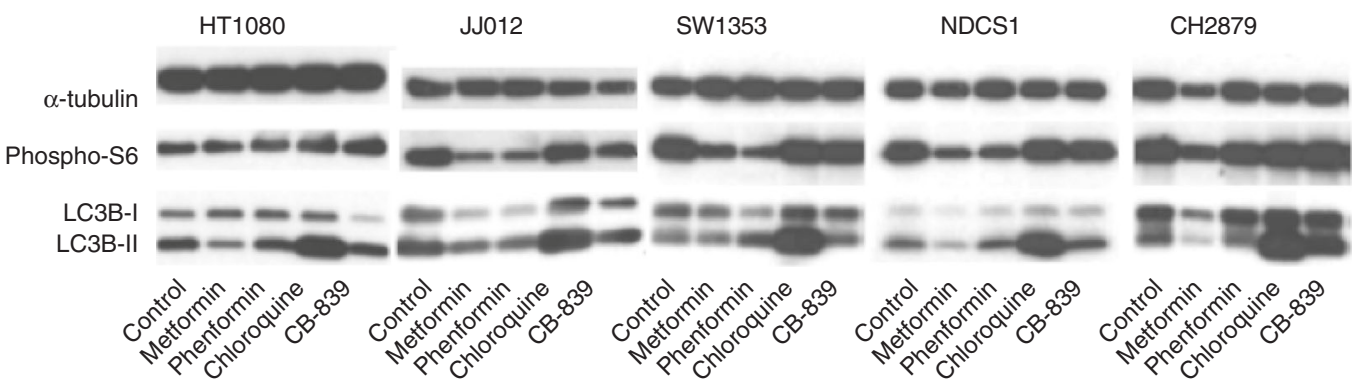

Fig. 5 The different compounds provoke different intracellular responses. a Caspase 3/7 activity of HT1080, JJ012, SW1353, NDCS1 and $\mathrm{CH} 2879$ cells after $24 \mathrm{~h}$ treatment with the metformin, phenformin, chloroquine and CB-839, as determined by caspase-glo $7 / 3$ assays. Only chloroquine slightly increases Caspase $3 / 7$ activity in 3/5 cell lines. $\mathrm{CH} 2879$ cells treated with ABT-737 and doxorubicin were used as positive control. For the negative control these compounds were combined with Z-vad-FMK. b Simultaneously to the measurement of caspase 3/7 activity, cell viability was measured using a Presto-Blue assay. c Western blot to evaluate the effect of metformin, phenformin, chloroquine and CB-839 on phosphorylated S6 and LC3B levels in five chondrosarcoma cell lines. Cell lines were treated for $72 \mathrm{~h}$ with their corresponding IC 50 values. Metformin and phenformin decreased levels of phosphorylated S6 in 4/5 and 3/5 cell lines, respectively, and decreased levels of LC3B in $4 / 5$ and $1 / 5$ cell lines, respectively. Chloroquine increased LC3B in all cell lines 
grade III chondrosarcomas) and the low-grade cartilage tumours (ACTs and enchondromas/osteochondromas) further demonstrated the significant difference in glutaminase expression levels between high-grade and low-grade cartilage tumours $(p<0.0001$, independent-samples $T$ test) (Fig. 2a). No difference in glutaminase expression between IDH1/2 mutant and $I D H 1 / 2$ wildtype central cartilage tumours was observed (Fig. 2b). Therefore, glutaminase is higher expressed in high-grade compared to lowgrade cartilage tumours but does not correlate to $I D H 1 / 2$ mutation status.

Using qRT-PCR analyses, we demonstrate that all cell lines have higher expression levels of glutaminase compared to the controls (growth plate and cartilage), although expression levels are variable (Fig. 3a). Inhibition of glutaminase using CB-839 in ten chondrosarcoma cell lines revealed that HT1080 $\left(I D H 1^{\mathrm{R} 132 \mathrm{C}}\right)$, SW1353 (IDH2 $\left.{ }^{\mathrm{R} 172 \mathrm{~S}}\right)$ and, to a lesser extent, JJ012 (IDH1 $\left.{ }^{\mathrm{R} 132 \mathrm{G}}\right)$, were very sensitive for glutaminase inhibition, with $I_{50}$ values below $5 \mu \mathrm{M}$ (Fig. 3b, Table 1). L2975 (IDH2 ${ }^{\mathrm{R} 172 \mathrm{~W}}$ ), NDCS1
$\left(\mathrm{IDH} 1 / 2^{\mathrm{WT}}\right)$ and $\mathrm{CH} 3573\left(\mathrm{IDH} 1 / 2^{\mathrm{WT}}\right)$ had $\mathrm{IC}_{50}$ values of $10.2,13.5$ and $17.5 \mu \mathrm{M}$, while the remaining four cell lines (one $I D H 1^{\mathrm{R} 132 \mathrm{C}}$, three $I D H 1 / 2^{\mathrm{WT}}$ ) had $\mathrm{IC}_{50}$ values above $50 \mu \mathrm{M}$. Interestingly, absence of FBS, increased the sensitivity to CB-839 especially in the $I D H 1 / 2$ mutant cell lines, while there was no clear difference in the cell lines with wildtype IDH1/2 (Fig. 3c). In conclusion, these experiments demonstrate that a subset of chondrosarcoma cell lines is dependent on glutaminase-mediated glutaminolysis to maintain cell viability.

Metformin, phenformin and chloroquine inhibit chondrosarcoma cell viability

Treating the chondrosarcoma cell line panel for $72 \mathrm{~h}$ with metformin, phenformin or chloroquine demonstrated that sensitivity for these compounds differed between the different chondrosarcoma cell lines (Fig. 4a). With an $\mathrm{IC}_{50}$ of $1.20 \mathrm{mM}$ and $17.1 \mu \mathrm{M}$ after $72 \mathrm{~h}$ of treatment, HT1080 cells have a higher sensitivity for respectively metformin and phenformin compared
A

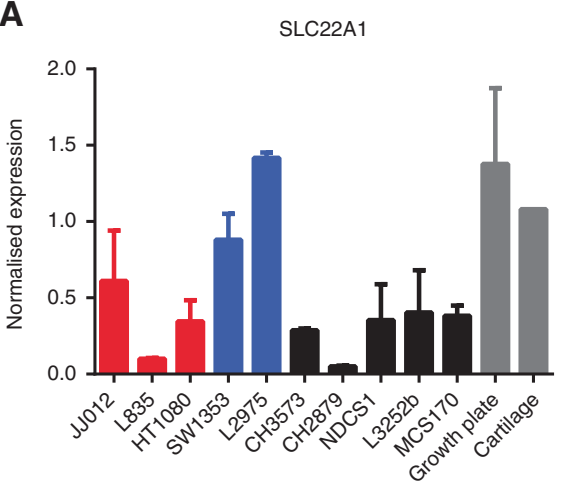

D

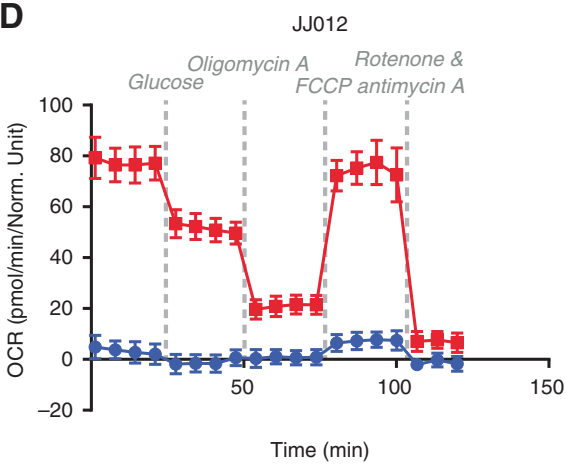

E

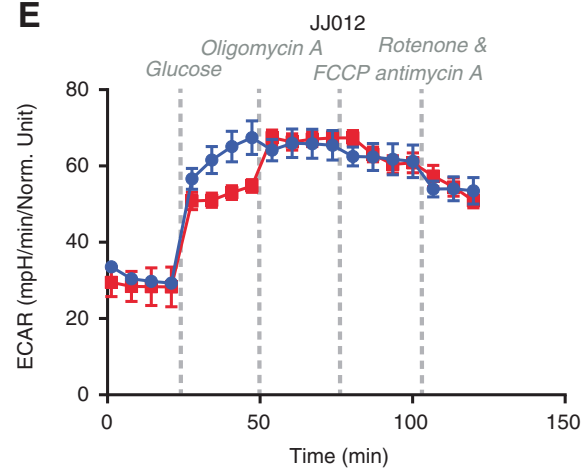

B
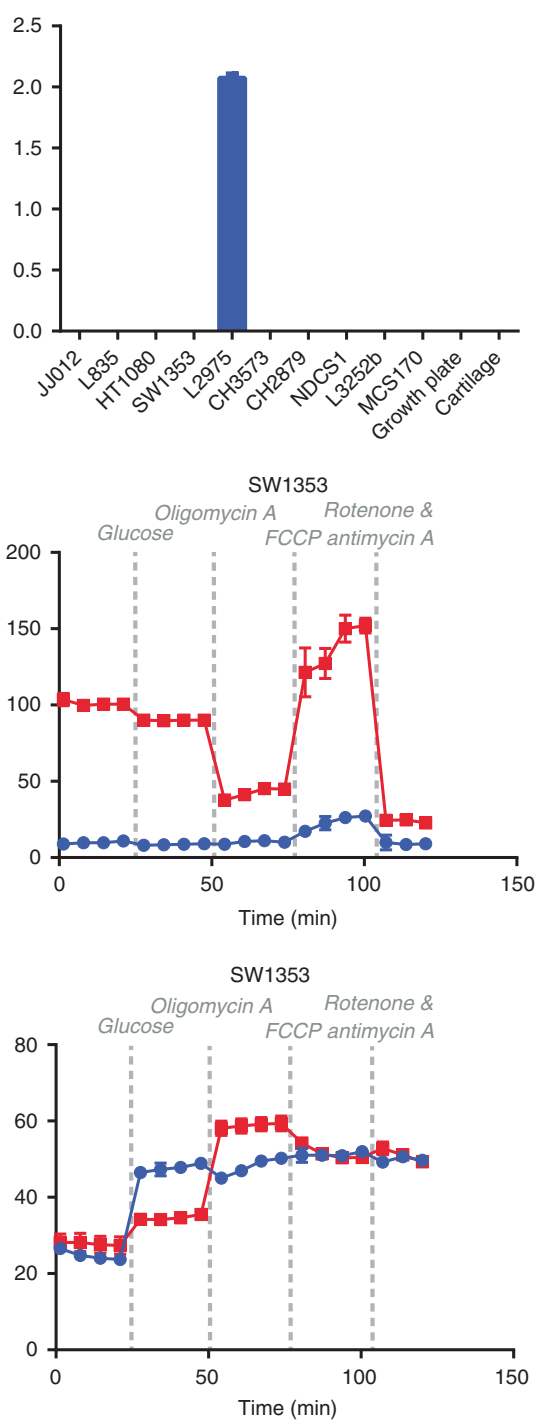
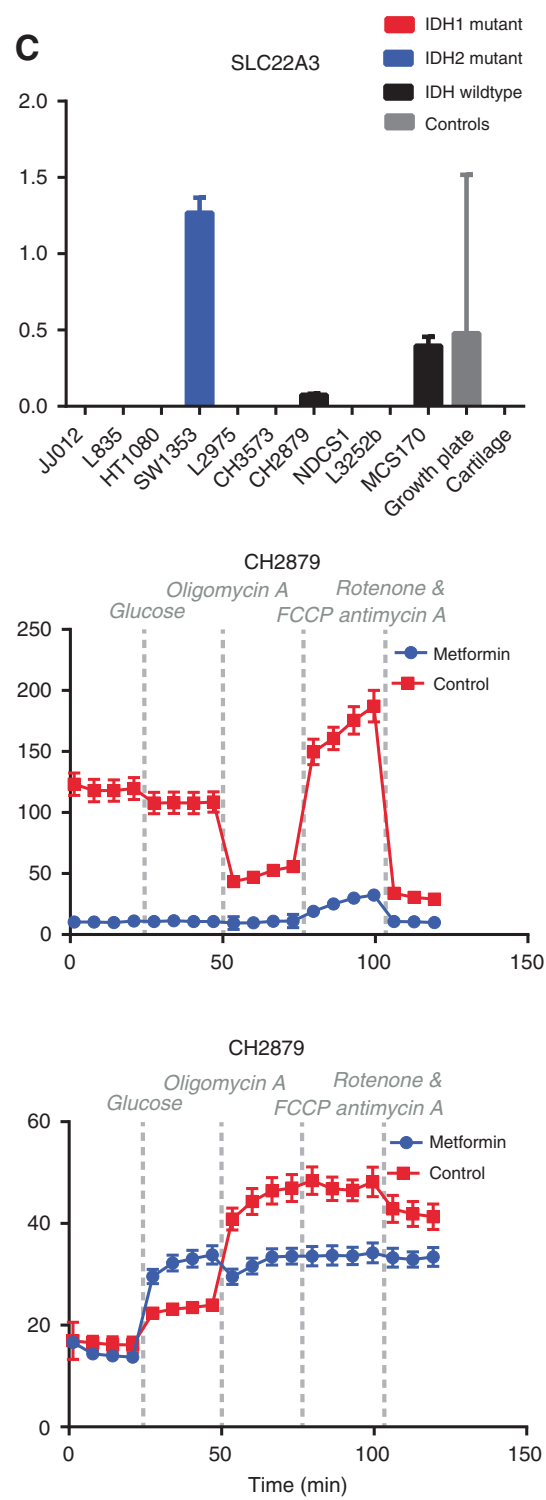

Fig. 6 Chondrosarcoma cells sufficiently express SLC22A1 for metformin to completely inhibit mitochondrial respiration. a All chondrosarcoma cell lines express SLC22A1. b SLC22A2 is only expressed by L2975. c Three out of ten chondrosarcoma cell lines express SLC22A3. d, e The impact of $24 \mathrm{~h}$ treatment with $5 \mathrm{mM}$ metformin on the Oxygen Consumption Rate (OCR)(D) and extracellular acidification rate (ECAR)(E) of JJ012, SW1353 and CH2879 was measured by Seahorse experiments. Metformin completely blocks mitochondrial respiration, which is only accompanied by a small increase in glycolysis 
to the other cell lines (Table 1). Treating the chondrosarcoma cell lines for 7 days increased the effect of metformin, phenformin and chloroquine on cell viability (Fig. 4b). Hoechst quantification confirmed that the effects on cell viability were caused by an absolute decrease in cell amount (Supplementary Figure 1). No difference between $I D H 1 / 2$ mutant and $I D H 1 / 2$ wildtype chondrosarcoma cell lines in sensitivity for metformin, phenformin and chloroquine was observed. To further demonstrate that the IDH1 mutant enzyme does not influence sensitivity to these compounds, the inhibitors were combined with AGI-5198, a specific inhibitor of the mutant IDH1 enzyme, in IDH1 mutant JJ012 and HT1080 cells. Cell viability (Fig. 4c and Supplementary Figure 2) was unaffected by cotreatment with AGI-5198. These results demonstrate that chondrosarcoma cell lines can be targeted by metformin, phenformin and chloroquine and further demonstrates the dependency of chondrosarcoma cell lines on glutaminolysis independent of the presence of the IDH1/2 mutant enzyme.

\section{Cellular effects of glutaminolysis inhibition}

To investigate the effect on apoptosis, caspase-glo 3/7 assays were performed. Chloroquine slightly increased caspase $3 / 7$ activity in three out of five cell lines tested (Fig. 5a). While the other compounds did impact cell viability after $24 \mathrm{~h}$ (Fig. 5b), no effect on caspase $3 / 7$ activity was observed. Next, we evaluated the effect of the four metabolic compounds on phosphorylated S6 protein levels, as this is an indicator of mTOR activity. As shown in Fig. 5c, metformin and phenformin decreased phosphorylated S6 levels in four out of five and three out of five cell lines respectively. However, metformin and phenformin did not affect phosphorylated S6 levels in HT1080 cells, the cell line that is most sensitive to metformin. Interestingly, metformin decreased LC3B-II levels in four out of five cell lines, which indicates an increase in autophagy. ${ }^{40}$ As expected, chloroquine greatly increased LC3B-II levels. CB-839 did not affect phosphorylated S6 or LC3B protein levels. Thus, while the induction of apoptosis is very limited, metformin and phenformin decreased mTOR activity in chondrosarcoma cells, and metformin decreased autophagy, an effect that is counteracted by chloroquine.

Metformin is sufficiently transported into chondrosarcoma cells to completely inhibit mitochondrial respiration

Next, expression levels of SLC22A1-3 were determined by qRTPCR analyses, as these transporters are essential for the cellular uptake of metformin and might explain the variability in sensitivity for metformin. Although expression is variable, all cell lines express SLC22A1 (Fig. 6a). Interestingly, the two IDH2 mutated cell lines had the highest expression of SLC22A1. SLC22A2 is only expressed by L2975 (Fig. 6b) and SLC22A3 is only expressed by SW1353, MCS170 and CH2879 (Fig. 6c). This demonstrates that all cell lines express transporters for the cellular uptake of metformin, but expression levels differ.

To evaluate the effect of metformin on chondrosarcoma cell metabolism, seahorse experiments with three chondrosarcoma cell lines (one IDH1 mutated, one IDH2 mutated and one IDH wildtype) were performed. Strikingly, metformin completely inhibited mitochondrial respiration in all cell lines tested independent of IDH1/2 mutation status or SLC22A1 levels (Fig. 6d). The small increase in OCR observed after the addition of FCCP and increased ECAR levels after glucose injection demonstrate that the metformin treated cells are still viable. Interestingly, metformin treated cells showed higher levels of glycolysis when glucose is present compared to the controls. However, oligomycin A injection increased glycolytic levels in controls but not in treated cells, indicating a maximum in glycolytic energy production is achieved in treated cells after glucose addition (Fig. 6e). The small difference in glycolytic activity between treated and untreated cells cannot compensate for the observed loss in ATP production through mitochondrial respiration, suggesting that chondrosarcoma cells utilise other pathways for energy production.

\section{DISCUSSION}

In this study, we demonstrated a difference in glutaminase expression levels between the different chondrosarcoma grades, with the highest expression observed in high-grade tumours. We therefore examined whether glutaminolysis could be exploited as a therapeutic target for high-grade chondrosarcoma. Based on current ongoing clinical trials targeting glutaminolysis, to which chondrosarcoma patients can be enrolled, we interfered with glutaminolysis in chondrosacoma cell lines at different levels (Fig. 1).

First, we inhibited glutaminase using the glutaminase inhibitor CB-839, and indeed, six out of ten chondrosarcoma cell lines showed $\mathrm{IC}_{50}$ values below $20 \mu \mathrm{M}$. CB-839 did not induce apoptosis, autophagy or mTOR activity, suggesting that it likely impacts cell viability via other mechanisms.

Second, we used the widely used anti-diabetic drug metformin, which, among other effects, indirectly inhibits glutaminase via cMyc; (Fig. 1), 21,22 inhibits complex 1 of the electron transport chain, ${ }^{20}$ and inhibits mTOR signalling. ${ }^{19}$ Indeed, a subset of chondrosarcoma cell lines was sensitive to metformin, especially when treated for a longer time period. mTOR signalling was previously shown to be important in chondrosarcoma ${ }^{41}$ and we confirmed that metformin decreased mTOR activity in all but one chondrosarcoma cell lines tested, which is in line with findings in other studies. ${ }^{19}$ Interestingly, mTOR activity was not inhibited in HT1080 which is the most sensitive for metformin treatment, suggesting that mTOR inhibition alone cannot explain the impact of metformin on chondrosarcoma cell viability. Furthermore, while apoptosis was absent, metformin seemed to induce autophagy in the majority of cell lines tested, which can likely be linked to the effect of metformin on AMPK. ${ }^{42}$ In this study, we further demonstrate that metformin completely blocks mitochondrial respiration in chondrosarcoma cells, likely caused by its effect on complex I of the electron transport chain. However, blocking complex I of the electron transfer chain (and therefore oxidative glutaminolysis) alone was not sufficient to inhibit cell viability, as an even higher concentration of metformin only had a small impact on cell viability within the 24-h time frame of the mitochondrial respiration experiments (Fig. 5b). The small difference in glycolysis observed upon treatment with metformin is likely insufficient to compensate for the total los of ATP production through oxidative means, suggesting that chondrosarcoma cells are not dependent on oxidative metabolism and require other sources, in addition to the increased glycolysis, to supply the necessary energy. In prostate cancer cell lines, it was demonstrated that metformin treatment increased the dependency on reductive glutaminolysis. ${ }^{43}$ Further identifying these pathways might provide interesting targets for combination treatment with metformin.

Third, as an alternative to metformin, we used its lipophilic analogue phenformin. In contrast to metformin, phenformin does not need SLC22A1-3 transport to get into cells.

As expected, the effect of phenformin on cell viability, mTOR activity, and apoptosis was very similar to metformin. Contrary to metformin, phenformin is not used in the clinic due to an increased risk of lactic acidosis. Moreover, we show that all chondrosarcoma cell lines sufficiently express the SLC22A1 transporter that is necessary for metformin uptake, suggesting that there is limited rationale to move to phenformin trials for chondrosarcomas when the metformin trial demonstrates limited efficacy.

Fourth, we evaluated the anti-malaria drug chloroquine, which, in addition to inhibiting glutamate dehydrogenase, is a wellknown inhibitor of autophagy and thereby inhibits many other 
metabolic and signal transduction pathways. ${ }^{44,45}$ The chondrosarcoma cell lines were sensitive to chloroquine. Also, we confirmed that chloroquine inhibited autophagy in all chondrosarcoma cell lines tested. Moreover, a slight induction of apoptosis was seen in three out of five chondrosarcoma cell lines.

Thus, we used four different drugs to evaluate whether the increased dependence on glutaminolysis could be therapeutically exploited using repurposing of existing drugs, and confirmed that a subset of chondrosarcoma cell lines is indeed sensitive to glutaminolysis inhibition. We could not identify a correlation between levels of glutaminase expression and sensitivity for any of these metabolic compounds in the panel of ten chondrosarcoma cell lines. Of note, there was also no correlation of the IDH1/ 2 mutation status of chondrosarcoma cells with sensitivity to these compounds, or with the expression levels of glutaminase in primary tumours. We could therefore not confirm the prevailing hypothesis that $I D H 1 / 2$ mutant chondrosarcoma cells rely on glutaminolysis to generate sufficient a-KG for $D-2-H G$ production, ${ }^{15-17}$ as it seems that also high-grade chondrosarcomas that are wildtype for $I D H 1 / 2$ depend on glutaminolysis. To explain this dependency of $I D H 1 / 2$ wildtype chondrosarcoma on glutaminolysis, it is tempting to speculate that the hypoxic microenvironment, which is a characteristic of chondrosarcoma, ${ }^{46}$ is equally important or even overrates the effect of the $I D H 1 / 2$ mutation in chondrosarcoma. HIF-1a activates pyruvate dehydrogenase kinase, which inactivates pyruvate dehydrogenase, thereby inhibiting the influx of pyruvate into the TCA cycle. ${ }^{47}$ In this context, TCA cycle anaplerosis is required for the synthesis of fatty acids, which is then primarily mediated via glutaminolysis. ${ }^{48}$ Therefore, the hypoxic microenvironment of chondrosarcomas potentially explains why chondrosarcomas depend on glutaminolysis irrespective of the presence or absence of an $I D H 1 / 2$ mutation.

Our results indicate that there is limited preclinical rationale to select chondrosarcoma patients for treatment with these compounds based on their IDH1/2 mutation status. This is in contrast to the studies by Cuyas et al. ${ }^{16}$ and Molenaar et al., ${ }^{49}$ where, respectively, an increased sensitivity for metformin was identified in an engineered IDH1 mutant breast cancer cell line, and in an engineered $I D H 1$ mutant colorectal cancer cell line compared to their wildtype parental cells. The differences in tumour types and the fact that these cell lines harbour an engineered instead of an endogenous IDH1/2 mutation likely explain the differences in experimental findings.

In conclusion, our results demonstrate that glutaminase is higher expressed in high-grade compared to low-grade chondrosarcomas. High-grade chondrosarcomas are dependent on glutaminolysis which is independent of $I D H 1 / 2$ mutation status. This dependence can be therapeutically exploited by repurposing existing drugs that inhibit glutaminolysis, including CB-839, metformin, phenformin and chloroquine.

\section{ACKNOWLEDGEMENTS}

The authors thank Dr. Joel A. Block (Rush University, Chicago, USA) for providing JJ012, Prof. Antonio Llombart-Bosch (Valencia University, Spain) for $\mathrm{CH} 2879$ and CH3573, Dr. M. Namba (Okayama University Medical School, Japan) for OUMS27, and Dr. Naoko Kudo (Niigata University Graduate School of Medical and Dental Sciences, Japan) for NDCS1. In addition, the authors thank Gaia Alberti, Hans Baelde, Pauline Wijers-Koster and Inge Briaire- de Bruijn for technical assistance, Jolieke G. van Oosterwijk for construction of the tissue microarray, and Willem Corver for help with the Seahorse experiments. This study was funded by the Leiden University Medical Center.

\section{AUTHOR CONTRIBUTIONS}

B.N., R.D.A., Y.dJ., A.B.K. and B.E.W.Mv.dA. performed the experiments. E.F.P.P. and A.H. G.C. performed all the scoring of the TMA'S. E.F.P.P., B.N., R.J.M., A.M.C.J. and J.V.M.G.B. designed the study. E.F.P.P., A.M.C.J. and J.V.M.G.B. supervised the experiments. E.F.P.
P. and J.V.M.G.B. wrote the manuscript. All authors read and approved the final version of the manuscript.

\section{ADDITIONAL INFORMATION}

Supplementary information is available for this paper at https://doi.org/10.1038/ s41416-018-0050-9.

Competing interests: The authors declare no competing interests.

Ethics approval and consent to participate: All samples were handled according to the Dutch code of proper secondary use of human material as accorded by the Dutch society of pathology (Federa). The samples were handled in a coded manner. All study methods were approved by the LUMC ethical board (B17.020 v2).

Availability of data and materials: The manuscript and the supplementary files contain all potential findings based on raw data analysis. Raw data can be obtained from authors on request.

Note: This work is published under the standard license to publish agreement. After 12 months the work will become freely available and the license terms will switch to a Creative Commons Attribution 4.0 International licence (CC BY 4.0).

\section{REFERENCES}

1. Hogendoorn, P. C. W., Bovée, J. V. M. G. \& Nielsen, G. P. in WHO Classification of Tumours of Soft Tissue and Bone (eds Fletcher, C. D. M., Bridge, J. A., Hogendoorn P. C. W. \& Mertens, F.) 264-268 (IARC, Lyon, 2013).

2. Gelderblom, H. et al. The clinical approach towards chondrosarcoma. Oncologist 13, 320-329 (2008).

3. Duchman, K. R., Lynch, C. F., Buckwalter, J. A. \& Miller, B. J. Estimated causespecific survival continues to improve over time in patients with chondrosarcoma. Clin. Orthop. Relat. Res. 472, 2516-2525 (2014).

4. Inwards, C. \& Hogendoorn, P. C. W. in WHO Classification of Tumours of Soft Tissue and Bone (eds Fletcher, C. D. M., Bridge, J. A., Hogendoorn P. C. W. \& Mertens, F.) 269-270 (IARC, Lyon, 2013).

5. Frezza, A. M. et al. Mesenchymal chondrosarcoma: prognostic factors and outcome in 113 patients. A European Musculoskeletal Oncology Society study. Eur. J. Cancer 51, 374-381 (2015).

6. Xu, J., Li, D., Xie, L., Tang, S. \& Guo, W. Mesenchymal chondrosarcoma of bone and soft tissue: a systematic review of 107 patients in the past 20 years. PLOS ONE. 10, e0122216 (2015)

7. Nakashima, Y., Pinieux, de, G. \& Ladanyi, M. in WHO Classification of Tumours of Soft Tissue and Bone (eds Fletcher, C. D. M., Bridge, J. A., Hogendoorn P. C. W. \& Mertens, F.) 271-272 (IARC, Lyon, 2013).

8. van Maldegem, A. M. et al. Outcome of advanced, unresectable conventional central chondrosarcoma. Cancer 120, 159-164 (2014).

9. Amary, M. F. et al. IDH1 and IDH2 mutations are frequent events in central chondrosarcoma and central and periosteal chondromas but not in other mesenchymal tumours. J. Pathol. 224, 334-343 (2011).

10. Meijer, D. et al. Genetic characterization of mesenchymal, clear cell, and dedifferentiated chondrosarcoma. Genes. Chromosomes Cancer 51, 899-909 (2012).

11. Amary, M. F. et al. Ollier disease and Maffucci syndrome are caused by somatic mosaic mutations of IDH1 and IDH2. Nat. Genet. 43, 1262-1265 (2011).

12. Pansuriya, T. C. et al. Somatic mosaic IDH1 and IDH2 mutations are associated with enchondroma and spindle cell hemangioma in Ollier disease and Maffucci syndrome. Nat. Genet. 43, 1256-1261 (2011).

13. $\mathrm{Xu}, \mathrm{W}$. et al. Oncometabolite 2-hydroxyglutarate is a competitive inhibitor of alpha-ketoglutarate-dependent dioxygenases. Cancer Cell 19, 17-30 (2011).

14. Suijker, J. et al. Inhibition of mutant IDH1 decreases D-2-HG levels without affecting tumorigenic properties of chondrosarcoma cell lines. Oncotarget 6 , 12505-12519 (2015).

15. Grassian, A. R. et al. IDH1 mutations alter citric acid cycle metabolism and increase dependence on oxidative mitochondrial metabolism. Cancer Res. 74, 3317-3331 (2014).

16. Cuyas, E. et al. Oncometabolic mutation IDH1 R132H confers a metforminhypersensitive phenotype. Oncotarget 6, 12279-12296 (2015).

17. Chen, R. et al. Hominoid-specific enzyme GLUD2 promotes growth of IDH1R132H glioma. Proc. Natl Acad. Sci. USA 111, 14217-14222 (2014).

18. Molenaar, R. J. et al. Study protocol of a phase IB/II clinical trial of metformin and chloroquine in patients with IDH1-mutated or IDH2-mutated solid tumours. BMJ Open 7, e014961 (2017). 
19. Jalving, M. et al. Metformin: taking away the candy for cancer? Eur. J. Cancer 46, 2369-2380 (2010)

20. Wheaton, W. W. et al. Metformin inhibits mitochondrial complex I of cancer cells to reduce tumorigenesis. elife 3, e02242 (2014).

21. Blandino, G. et al. Metformin elicits anticancer effects through the sequential modulation of DICER and c-MYC. Nat. Commun. 3, 865 (2012).

22. Gao, P. et al. c-Myc suppression of miR-23a/b enhances mitochondrial glutaminase expression and glutamine metabolism. Nature 458, 762-765 (2009).

23. Yee, S. W. et al. Prediction and validation of enzyme and transporter off-targets for metformin. J. Pharmacokinet. Pharmacodyn. 42, 463-475 (2015).

24. Jarzyna, R., Lenarcik, E. \& Bryla, J. Chloroquine is a potent inhibitor of glutamate dehydrogenase in liver and kidney-cortex of rabbit. Pharmacol. Res. 35, 79-84 (1997).

25. Choi, M. M. et al. Inhibitory properties of nerve-specific human glutamate dehydrogenase isozyme by chloroquine. J. Biochem. Mol. Biol. 40, 1077-1082 (2007).

26. van Oosterwijk, J. G. et al. Src kinases in chondrosarcoma chemoresistance and migration: dasatinib sensitises to doxorubicin in TP53 mutant cells. Br. J. Cancer 109, 1214-1222 (2013).

27. Baranski, Z. et al. Aven-mediated checkpoint kinase control regulates proliferation and resistance to chemotherapy in conventional osteosarcoma. J. Pathol. 236, 348-359 (2015).

28. Scully, S. P. et al. Marshall Urist Award. Interstitial collagenase gene expression correlates with in vitro invasion in human chondrosarcoma. Clin. Orthop. Relat. Res. 376, 291-303 (2000).

29. van Oosterwijk, J. G. et al. Three new chondrosarcoma cell lines: one grade III conventional central chondrosarcoma and two dedifferentiated chondrosarcomas of bone. BMC Cancer 12, 375 (2012).

30. Rasheed, S., Nelson-Rees, W. A., Toth, E. M., Arnstein, P. \& Gardner, M. B. Characterization of a newly derived human sarcoma cell line (HT-1080). Cancer 33, 1027-1033 (1974).

31. Gil-Benso, R. et al. Establishment and characterization of a continuous human chondrosarcoma cell line, ch-2879: comparative histologic and genetic studies with its tumor of origin. Lab. Invest. 83, 877-887 (2003).

32. de Jong, Y. et al. Inhibition of Bcl-2 family members sensitizes mesenchymal chondrosarcoma to conventional chemotherapy: report on a novel mesenchymal chondrosarcoma cell line. Lab. Invest. 96, 1128-1137 (2016).

33. Calabuig-Farinas, S. et al. Characterization of a new human cell line $(\mathrm{CH}-3573)$ derived from a grade II chondrosarcoma with matrix production. Pathol. Oncol. Res. 18, 793-802 (2012).

34. Kudo, N. et al. Establishment of novel human dedifferentiated chondrosarcoma cell line with osteoblastic differentiation. Virchows Arch. 451, 691-699 (2007).
35. Martins-Neves SR, et al. Osteosarcoma Stem Cells Have Active Wnt/beta-catenin and Overexpress SOX2 and KLF4. Journal of cellular physiology 2016;231(4):87686.

36. Rozeman, L. B. et al. Absence of $\mathrm{IHH}$ and retention of PTHrP signalling in enchondromas and central chondrosarcomas. J. Pathol. 205, 476-482 (2005).

37. Hameetman, L. et al. Peripheral chondrosarcoma progression is accompanied by decreased Indian Hedgehog signalling. J. Pathol. 209, 501-511 (2006).

38. van Oosterwijk, J. G. et al. Restoration of chemosensitivity for doxorubicin and cisplatin in chondrosarcoma in vitro: BCL-2 family members cause chemoresistance. AnnOncol 23, 1617-1626 (2012).

39. de Jong, Y. et al. Targeting survivin as a potential new treatment for chondrosarcoma of bone. Oncogenesis 5, e222 (2016).

40. Barth, S., Glick, D. \& Macleod, K. F. Autophagy: assays and artifacts. J. Pathol. 221, 117-124 (2010).

41. Zhang, Y. X. et al. Functional profiling of receptor tyrosine kinases and downstream signaling in human chondrosarcomas identifies pathways for rational targeted therapy. Clin. Cancer Res. 19, 3796-3807 (2013).

42. Kim, J., Kundu, M., Viollet, B. \& Guan, K. L. AMPK and mTOR regulate autophagy through direct phosphorylation of Ulk1. Nat. Cell Biol. 13, 132-141 (2011).

43. Fendt, S. M. et al. Metformin decreases glucose oxidation and increases the dependency of prostate cancer cells on reductive glutamine metabolism. Cancer Res. 73, 4429-4438 (2013).

44. Reyjal, J., Cormier, K. \& Turcotte, S. Autophagy and cell death to target cancer cells: exploiting synthetic lethality as cancer therapies. Adv. Exp. Med. Biol. 772, 167-188 (2014).

45. Pascolo, S. Time to use a dose of Chloroquine as an adjuvant to anti-cancer chemotherapies. Eur. J. Pharmacol. 771, 139-144 (2016).

46. Boeuf, S., Bovee, J. V., Lehner, B., Hogendoorn, P. C. \& Richter, W. Correlation of hypoxic signalling to histological grade and outcome in cartilage tumours. Histopathology 56, 641-651 (2010).

47. Kim, J. W., Tchernyshyov, I., Semenza, G. L. \& Dang, C. V. HIF-1-mediated expression of pyruvate dehydrogenase kinase: a metabolic switch required for cellular adaptation to hypoxia. Cell Metab. 3, 177-185 (2006).

48. Wise, D. R. et al. Hypoxia promotes isocitrate dehydrogenase-dependent carboxylation of alpha-ketoglutarate to citrate to support cell growth and viability. Proc. Natl Acad. Sci. USA 108, 19611-19616 (2011).

49. Molenaar, R. J. et al. Radioprotection of IDH1-mutated cancer cells by the IDH1mutant inhibitor AGI-5198. Cancer Res. 75, 4790-4802 (2015). 